\title{
Existence of Solutions for the Discrete Dirichlet Problem Involving $p$-Mean Curvature Operator
}

\author{
Jianxia Wang $\mathbb{D}^{1,2}$ and Zhan Zhou $\mathbb{D}^{1,2}$ \\ ${ }^{1}$ School of Mathematics and Information Science, Guangzhou University, Guangzhou 510006, China \\ ${ }^{2}$ Center for Applied Mathematics, Guangzhou University, Guangzhou 510006, China \\ Correspondence should be addressed to Zhan Zhou; zzhou0321@hotmail.com
}

Received 4 July 2020; Accepted 27 July 2020; Published 29 August 2020

Academic Editor: Abdul Qadeer Khan

Copyright (c) 2020 Jianxia Wang and Zhan Zhou. This is an open access article distributed under the Creative Commons Attribution License, which permits unrestricted use, distribution, and reproduction in any medium, provided the original work is properly cited.

This work is to discuss the Dirichlet boundary value problem of the difference equation with $p$-mean curvature operator. Under some determinate growth conditions on the nonlinear term, the existence of one solution or two nontrivial solutions is obtained via variational methods and some analysis techniques. Examples are also given to illustrate our theorems in the last section.

\section{Introduction}

Let $\mathbb{N}, \mathbb{Z}$, and $\mathbb{R}$ stand for the sets of natural numbers, integer numbers, and real numbers, respectively. For $m, n \in \mathbb{Z}$, define $\mathbb{Z}[m,+\infty)=\{m, m+1, \ldots\} \quad$ and $\mathbb{Z}[m, n]=\{m, m+1, \ldots, n\}$ when $m \leq n$.

Consider the following Dirichlet boundary value problem:

$$
\left\{\begin{array}{l}
-\Delta\left(\phi_{p, c}(\Delta u(j-1))\right)=f(j, u(j)), \quad j \in \mathbb{Z}[1, b], \\
u(0)=u(b+1)=0,
\end{array}\right.
$$

where $b$ is a given positive integer, $f(j, \cdot) \in C(\mathbb{R}, \mathbb{R})$ for each $j \in \mathbb{Z}[1, b], \Delta$ is the forward difference operator defined by $\Delta u(j)=u(j+1)-u(j)$, and $\phi_{p, c}(t):=\left(1+|t|^{2}\right)^{(p-2) / 2} t$, $p \in(0,+\infty)$. Here, $\phi_{p, c}(\Delta u(j-1))$ is counted as a discretization of $p$-mean curvature operator.

We may regard problem (1) as being a discrete analog of the following differential problem in one-dimensional space:

$$
\begin{cases}-\operatorname{div}\left(\phi_{p, c}(\nabla u)\right)=f(x, u), & x \in \Omega \subset \mathbb{R}^{n} \\ u=0, & x \in \partial \Omega\end{cases}
$$

where $\phi_{p, c}(\nabla u)$, named $p$-mean curvature operator [1], is a generalization of mean curvature operator ([2], page 1212). When $p=1$, it reduces to mean curvature operator [3-6]. When $p=2$, it reduces to Laplacian operator.

Difference equations arise in different research fields, for instance, computer science, mechanical engineering, control systems, artificial or biological neural networks, economics, and so on, see [7-12].

In order to research discrete boundary problems, different methods have been used: fixed point theorems, upper and lower solutions techniques, see $[3,5]$ and the references given therein. Starting from Guo and Yu [13], various results have been achieved via variational methods, see [14-32].

In 2011, Candito and Bisci [33] studied the boundary value problem:

$$
\left\{\begin{array}{l}
-\Delta^{2} u(j-1)=f(j, u(j)), \quad j \in \mathbb{Z}[1, b], \\
u(0)=u(b+1)=0,
\end{array}\right.
$$

where $\Delta^{2} u(j)=\Delta(\Delta u(j)), f(j, t)$ is continuous with respect to $t$ for every $j \in \mathbb{Z}[1, b]$. Through variational methods, they proved that problem (3) admits two nontrivial solutions.

In 2015, Zhou and $\mathrm{Su}$ [30] discussed the following problem: 


$$
\begin{cases}\Delta^{n}\left(r(j-n) \phi_{c}\left(\Delta^{n} u(j-n)\right)\right)+(-1)^{n+1} f(j, u(j+1), u(j), u(j-1))=0, & j \in \mathbb{Z}[1, b], \\ u(j)=0, & j \in \mathbb{Z}[1-n, 0] \cup \mathbb{Z}[b+1, b+n],\end{cases}
$$

where $b \geq 2$ is positive integer, $\Delta^{m} u(j)=\Delta\left(\Delta^{m-1} u(j)\right)$, and $\phi_{c}(t)=t / \sqrt{1+|t|^{2}}$. By critical point theory and some analytical skills, two nontrivial solutions were acquired.

Motivated by the works of $[15,30,34-43]$, we deal with problem (1). Compared to problem (4), problem (1) is much more difficult to handle because of $p$-mean curvature operator. It needs more analytical skills. For the convenience of analysis, we have no option but to split the problem into two types: $0<p<2$ and $2 \leq p<+\infty$.

This paper is arranged as follows. In Section 2, we establish an appropriate Banach space and a suitable functional corresponding to problem (1). In order to gain solutions of problem (1), some preliminary knowledge is introduced. In Section 3, under certain growth conditions on the antiderivative $F$ of the nonlinearity $f$, one solution or two nontrivial solutions for problem (1) are obtained. In Section 4 , we give two concrete examples to demonstrate the applicability of our main results.

\section{Mathematical Background}

For problem (1), we naturally select the $b$-dimensional Banach space:

$$
S=\{u: \mathbb{Z}[0, b+1] \longrightarrow \mathbb{R}: u(0)=u(b+1)=0\},
$$

equipped with the norm

$$
\|u\|:=\left(\sum_{j=1}^{b}(u(j))^{2}\right)^{1 / 2}
$$

for all $u \in S$.

Its infinity norm is

$$
\|u\|_{\infty}:=\max _{j \in \mathbb{Z}[1, b]}|u(j)|, \quad \text { for all } u \in S .
$$

Apparently, one has

$$
\|u\|_{\infty} \leq\|u\|
$$

Furthermore, we need to use the following inequality. When $0<r<s, x_{j} \geq 0, j \in \mathbb{Z}[1, n]$, one has

$$
\left(\sum_{j=1}^{n} x_{j}^{s}\right)^{1 / s} \leq\left(\sum_{j=1}^{n} x_{j}^{r}\right)^{1 / r}
$$

see ([44], page 28).

Putting

$$
F(j, t):=\int_{0}^{t} f(j, \tau) \mathrm{d} \tau,
$$

the function $I: S \longrightarrow \mathbb{R}$ is defined by

$$
I(u):=\Phi(u)-\Psi(u),
$$

for every $u \in S$, where

$$
\begin{aligned}
& \Phi(u):=\frac{1}{p} \sum_{j=0}^{b}\left(\left(1+(\Delta u(j))^{2}\right)^{p / 2}-1\right), \\
& \Psi(u):=\sum_{j=1}^{b} F(j, u(j)) .
\end{aligned}
$$

It is not difficult to verify that $I \in C^{1}(S, \mathbb{R})$, and the critical points of $I$ on $S$ are exactly the solutions of problem (1).

One can see that

$$
\begin{aligned}
\sum_{j=0}^{b}(\Delta u(j))^{2} & =\sum_{j=0}^{b}(u(j+1)-u(j))^{2} \\
& =2 \sum_{j=1}^{b}(u(j))^{2}-2 \sum_{j=1}^{b-1} u(j+1) u(j) \\
& =\tilde{u} A \tilde{u}^{t r},
\end{aligned}
$$

where $\tilde{u}=(u(1), u(2), \ldots, u(b))$ is a $b$-dimensional row vector, $\widetilde{u}^{t r}$ stands for the transpose of $\widetilde{u}$, and

$$
A=\left(\begin{array}{ccccc}
2 & -1 & 0 & \cdots & 0 \\
-1 & 2 & -1 & \cdots & 0 \\
\vdots & \vdots & \vdots & \vdots & \vdots \\
0 & \cdots & -1 & 2 & -1 \\
0 & \cdots & 0 & -1 & 2
\end{array}\right)_{b \times b}
$$

Matrix $A$ is symmetric and positive definite. It is easy to see that $A$ has $b$ distinct positive eigenvalues which are given by

$$
\lambda_{j}:=4 \sin ^{2}\left(\frac{j \pi}{2(b+1)}\right), \quad \forall j \in \mathbb{Z}[1, b] .
$$

We have the following lemma, see ([36], page 918).

Lemma 1. For every $u \in S$, one has

$$
\lambda_{1}\|u\|^{2} \leq \sum_{j=0}^{b}(\Delta u(j))^{2} \leq \lambda_{b}\|u\|^{2}
$$

Let $E$ be a real Banach space, the functional $I \in C^{1}(E, \mathbb{R})$ is said to satisfy the Palais-Smale (P. S. for short) condition if any sequence $\left\{u_{n}\right\}$ in $E$ such that $I\left(u_{n}\right)$ is bounded and $I^{\prime}\left(u_{n}\right) \longrightarrow 0$ as $n \longrightarrow \infty$ contains a convergent subsequence.

Let $B_{r}$ denote the open ball in $E$ of radius $r$ about 0 , and let $\partial B_{r}$ denote its boundary.

Our main tool is Mountain pass lemma, see ([45], Theorem 2.2).

Lemma 2. Let $E$ be a real Banach space and $I \in C^{1}(E, \mathbb{R})$ satisfying the $P$. S. condition. Suppose $I(0)=0$ and

(i) There are constants $\rho, \alpha>0$ such that $I_{\partial B_{\rho}} \geq \alpha$ 
(ii) There exists an $e \in E / \bar{B}_{\rho}$ such that $I(e) \leq 0$

Then, I admits a critical value $c \geq \alpha$. In addition, $c$ can be characterized as

$$
c=\inf _{\gamma \in \Gamma} \max _{u \in \gamma([0,1])} I(u)
$$

where

$$
\Gamma=\{\gamma \in C([0,1], E) \mid \gamma(0)=0, \gamma(1)=e\} .
$$

\section{Main Results and Their Proofs}

Theorem 1. Let $p \in(0,2)$, for every $j \in \mathbb{Z}[1, b], f(j, \cdot)$ : $\mathbb{R} \longrightarrow \mathbb{R}$ be continuous. Suppose that $\left(F_{1}\right) \lim \sup _{t \rightarrow \infty} F$ $(j, t) /|t|^{p}<b^{(p / 2)-1} / p(2 \sin (\pi / 2(b+1)))^{p}, \forall j \in \mathbb{Z}[1, b]$.

Then, $I$ is coercive, and problem (1) admits at least one solution.

Proof. It is obvious that $I \in C^{1}(S, \mathbb{R})$.

Owing to $\left(F_{1}\right)$, we choose

$$
l_{1} \in\left(0, \frac{b^{p / 2-1}}{p}\left(2 \sin \left(\frac{\pi}{2(b+1)}\right)\right)^{p}\right)
$$

and $M_{1}>0$ such that, for every $j \in \mathbb{Z}[1, b]$ and $|t|>M_{1}$, $F(j, t)<l_{1}|t|^{p}$. Since $t \longrightarrow F(j, t)-l_{1}|t|^{p}$ is continuous on $\left[-M_{1}, M_{1}\right]$, there exists some constant $C_{1}>0$ such that $F(j, t)-l_{1}|t|^{p} \leq C_{1}$, for all $(j, t) \in \mathbb{Z}[1, b] \times\left[-M_{1}, M_{1}\right]$. Consequently, we follow that

$$
F(j, t) \leq l_{1}|t|^{p}+C_{1},
$$

for $\operatorname{every}(j, t) \in \mathbb{Z}[1, b] \times \mathbb{R}$.

Using (9) and (20), Hölder inequality, and Lemma 1, we follow that

$$
\begin{aligned}
I(u)= & \frac{1}{p} \sum_{j=0}^{b}\left(1+(\Delta u(j))^{2}\right)^{p / 2}-\sum_{j=1}^{b} F(j, u(j))-\frac{b+1}{p} \\
\geq & \frac{1}{p} \sum_{j=0}^{b}\left((\Delta u(j))^{2}\right)^{p / 2}-l_{1} \sum_{j=1}^{b}|u(j)|^{p}-\frac{b+1}{p}-C_{1} b \\
\geq & \frac{1}{p}\left(\sum_{j=0}^{b}(\Delta u(j))^{2}\right)^{p / 2}-l_{1} \sum_{j=1}^{b}|u(j)|^{p}-\frac{b+1}{p}-C_{1} b \\
\geq & \frac{1}{p}\left(\lambda_{1}\|u\|^{2}\right)^{p / 2}-l_{1} b^{1-(p / 2)}\|u\|^{p}-\frac{b+1}{p}-C_{1} b \\
= & b^{1-(p / 2)}\left(\frac{b^{(p / 2)-1}}{p}\left(2 \sin \left(\frac{\pi}{2(b+1)}\right)\right)^{p}-l_{1}\right)\|u\|^{p} \\
& -\frac{b+1}{p}-C_{1} b .
\end{aligned}
$$

Note that

$$
\frac{b^{(p / 2)-1}}{p}\left(2 \sin \left(\frac{\pi}{2(b+1)}\right)\right)^{p}-l_{1}>0
$$

and we have $\lim _{\|u\| \longrightarrow+\infty} I(u)=+\infty$. So, $I$ is coercive in S. Hence, $I$ is bounded from below. Set $\bar{c}=\inf _{u \in S} I(u)$. According to the coercivity of the functional $I$ again, there is $L>0$ such that $I(u)>\bar{c}+1$, for every $\|u\|>L$. Thus, $\inf _{\|u\| \leq L} I(u)=\inf _{u \in S} I(u)$. Taking into account that $S$ is a finite dimensional space and $I$ is continuous, $I$ possesses a critical point $\bar{u} \in S$ satisfying $I(\bar{u})=\inf _{u \in S} I(u)$. The proof is completed.

Theorem 2. Let $p \in(0,2)$, for every $j \in \mathbb{Z}[1, b]$, $f(j, \cdot): \mathbb{R} \longrightarrow \mathbb{R}$ be continuous. Suppose that

$$
\left(F_{2}\right) \lim _{t \rightarrow \infty} \inf \frac{F(j, t)}{|t|^{p}}>\frac{(b+1)^{1-(p / 2)}}{p}\left(2 \sin \left(\frac{b \pi}{2(b+1)}\right)\right)^{p} \text {, }
$$

$\forall j \in \mathbb{Z}[1, b]$.

Then, $I$ is anticoercive, and problem (1) has at least one solution.

Proof. To avoid repetition, we give only the key steps of the proof.

By $\left(F_{2}\right)$, we choose

$$
l_{2} \in\left(\frac{(b+1)^{1-(p / 2)}}{p}\left(2 \sin \left(\frac{b \pi}{2(b+1)}\right)\right)^{p},+\infty\right)
$$

and $C_{2}>0$ such that

$$
F(j, t) \geq l_{2}|t|^{p}-C_{2},
$$

for every $(j, t) \in \mathbb{Z}[1, b] \times \mathbb{R}$.

By virtue of (9) and (25), Hölder inequality, and Lemma 1, we follow that

$$
\begin{aligned}
I(u) & =\frac{1}{p} \sum_{j=0}^{b}\left(\left(1+(\Delta u(j))^{2}\right)^{p / 2}-1\right)-\sum_{j=1}^{b} F(j, u(j)) \\
& \leq \frac{1}{p} \sum_{j=0}^{b}\left((\Delta u(j))^{2}\right)^{p / 2}-l_{2} \sum_{j=1}^{b}|u(k)|^{p}+C_{2} b \\
& \leq \frac{1}{p}(b+1)^{1-(p / 2)}\left(\sum_{j=0}^{b}(\Delta u(j))^{2}\right)^{p / 2}-l_{2}\|u\|^{p}+C_{2} b \\
& \leq\left(\frac{(b+1)^{1-(p / 2)}}{p}\left(\lambda_{b}\right)^{p / 2}-l_{2}\right)\|u\|^{p}+C_{2} b \\
& =\left(\frac{(b+1)^{1-(p / 2)}}{p}\left(2 \sin \left(\frac{b \pi}{2(b+1)}\right)\right)^{p}-l_{2}\right)\|u\|^{p}+C_{2} b .
\end{aligned}
$$


Since

$$
\frac{(b+1)^{1-(p / 2)}}{p}\left(2 \sin \left(\frac{b \pi}{2(b+1)}\right)\right)^{p}-l_{2}<0,
$$

we have $\lim _{\|u\| \rightarrow+\infty} I(u)=-\infty$.

Theorem 3. Let $p \in[2,+\infty)$, for every $j \in \mathbb{Z}[1, b]$, $f(j, \cdot): \mathbb{R} \longrightarrow \mathbb{R}$ be continuous. Suppose that

$\left(F_{3}\right) \lim _{t \longrightarrow \infty} \sup \frac{F(j, t)}{|t|^{p}}<\frac{(b+1)^{1-(p / 2)}}{p}\left(2 \sin \left(\frac{\pi}{2(b+1)}\right)\right)^{p}$,

$\forall j \in \mathbb{Z}[1, b]$.

Then, $I$ is coercive, and problem (1) possesses at least one solution.

Proof. Here, we give only the main steps of the proof.

By $\left(F_{3}\right)$, we choose

$$
l_{3} \in\left(0, \frac{(b+1)^{1-(p / 2)}}{p}\left(2 \sin \left(\frac{\pi}{2(b+1)}\right)\right)^{p}\right)
$$

and $C_{3}>0$ such that

$$
F(j, t) \leq l_{3}|t|^{p}+C_{3},
$$

for every $(j, t) \in \mathbb{Z}[1, b] \times \mathbb{R}$.

Thanks to (9) and (30), Hölder inequality, and Lemma 1 , and we follow that

$$
\begin{aligned}
I(u) & =\frac{1}{p} \sum_{j=0}^{b}\left(\left(1+(\Delta u(j))^{2}\right)^{p / 2}-1\right)-\sum_{j=1}^{b} F(j, u(j)) \\
& \geq \frac{1}{p} \sum_{j=0}^{b}\left((\Delta u(j))^{2}\right)^{p / 2}-l_{3} \sum_{j=1}^{b}|u(j)|^{p}-C_{3} b \\
& \geq \frac{1}{p}(b+1)^{1-(p / 2)}\left(\sum_{j=0}^{b}(\Delta u(j))^{2}\right)^{p / 2}-l_{3}\|u\|^{p}-C_{3} b \\
& \geq\left(\frac{(b+1)^{1-(p / 2)}}{p}\left(\lambda_{1}\right)^{p / 2}-l_{3}\right)\|u\|^{p}-C_{3} b \\
& =\left(\frac{(b+1)^{1-(p / 2)}}{p}\left(2 \sin \left(\frac{\pi}{2(b+1)}\right)\right)^{p}-l_{3}\right)\|u\|^{p}-C_{3} b .
\end{aligned}
$$

Since

$$
\frac{(b+1)^{1-(p / 2)}}{p}\left(2 \sin \left(\frac{\pi}{2(b+1)}\right)\right)^{p}-l_{3}>0
$$

we have

$$
\lim _{\|u\| \rightarrow+\infty} I(u)=+\infty
$$

Theorem 4. Let $p \in[2,+\infty)$, for every $j \in \mathbb{Z}[1, b]$, $f(j, \cdot): \mathbb{R} \longrightarrow \mathbb{R}$ be continuous. Suppose that

$$
\left(F_{4}\right) \lim _{t \rightarrow \infty} \inf \frac{F(j, t)}{|t|^{p}}>\frac{b^{(p / 2)-1}}{p}\left(2 \sin \left(\frac{b \pi}{2(b+1)}\right)\right)^{p},
$$

$$
\forall j \in \mathbb{Z}[1, b] .
$$

Then, $I$ is anticoercive, and problem (1) admits at least one solution.

Proof. In the same way, we give only the crucial steps of the proof.

By $\left(F_{4}\right)$, we choose

$$
l_{4} \in\left(\frac{b^{(p / 2)-1}}{p}\left(2 \sin \left(\frac{b \pi}{2(b+1)}\right)\right)^{p},+\infty\right)
$$

and $C_{4}>0$ such that

$$
F(j, t) \geq l_{4}|t|^{p}-C_{4},
$$

for every $(j, t) \in \mathbb{Z}[1, T] \times \mathbb{R}$.

Given any $u \neq 0$, applying (9) and (36), Hölder inequality, and Lemma 1 , we follow that

$$
\begin{aligned}
I(u)= & \frac{1}{p} \sum_{j=0}^{b}\left(1+(\Delta u(j))^{2}\right)^{p / 2}-\sum_{j=1}^{b} F(j, u(j))-\frac{b+1}{p} \\
\leq & \frac{1}{p}\left(\sum_{j=0}^{b}\left(1+(\Delta u(j))^{2}\right)\right)^{p / 2}-l_{4} \sum_{j=1}^{b}|u(j)|^{p}-\frac{b+1}{p}+C_{4} b \\
\leq & \frac{1}{p}\left(b+1+\lambda_{b}\|u\|^{2}\right)^{p / 2}-l_{4} b^{1-(p / 2)}\|u\|^{p}-\frac{b+1}{p}+C_{4} b \\
\leq & b^{1-(p / 2)}\left(\frac{b^{(p / 2)-1}}{p}\left(\frac{b+1}{\|u\|^{2}}+\lambda_{b}\right)^{p / 2}-l_{4}\right)\|u\|^{p}-\frac{b+1}{p}+C_{4} b \\
= & b^{1-(p / 2)}\left(\frac{b^{(p / 2)-1}}{p}\left(\frac{b+1}{\|u\|^{2}}+4 \sin ^{2}\left(\frac{b \pi}{2(b+1)}\right)\right)^{p / 2}-l_{4}\right) \\
& \cdot\|u\|^{p}-\frac{b+1}{p}+C_{4} b .
\end{aligned}
$$

Note that

$$
\begin{aligned}
& \lim _{\|u\| \rightarrow+\infty}\left(\frac{b^{(p / 2)-1}}{p}\left(\frac{b+1}{\|u\|^{2}}+4 \sin ^{2}\left(\frac{b \pi}{2(b+1)}\right)\right)^{p / 2}-l_{4}\right) \\
& =\frac{b^{p / 2}-1}{p}\left(2 \sin \left(\frac{b \pi}{2(b+1)}\right)\right)^{p}-l_{4}<0,
\end{aligned}
$$

we have

$$
\lim _{\|u\| \rightarrow+\infty} I(u)=-\infty .
$$


Remark 1. If moreover, in Theorems $1-4$, if $f(j, 0) \neq 0$ for some $j \in \mathbb{Z}[1, b]$, then the solution is nontrivial.
Corollary 1. Let $p \in(0,2)$, for every $j \in \mathbb{Z}[1, b]$, $f(j, \cdot): \mathbb{R} \longrightarrow \mathbb{R}$ be continuous, and satisfy one of the following conditions:

$$
\begin{aligned}
& \left(f_{1}\right) \lim _{t \rightarrow \infty} \sup \frac{f(j, t)}{|t|^{p-2} t}<b^{(p / 2)-1}\left(2 \sin \left(\frac{\pi}{2(b+1)}\right)\right)^{p}, \quad \forall j \in \mathbb{Z}[1, b], \\
& \left(f_{2}\right) \lim _{t \rightarrow \infty} \inf \frac{f(j, t)}{|t|^{p-2} t}>(b+1)^{1-(p / 2)}\left(2 \sin \left(\frac{b \pi}{2(b+1)}\right)\right)^{p}, \quad \forall j \in \mathbb{Z}[1, b] .
\end{aligned}
$$

Then, problem (1) has at least one solution.

Proof. Here, we only prove that $\left(f_{2}\right)$ implies $\left(F_{2}\right)$. The proof that $(f)_{1}$ implies $\left(F_{1}\right)$ refers to ([33], Corollary 2.1).

From $\left(f_{2}\right)$, there exist $\varepsilon>0$ and $L>0$ such that

$$
\frac{f(j, t)}{|t|^{p-2} t}>(b+1)^{1-(p / 2)}\left(2 \sin \left(\frac{b \pi}{2(b+1)}\right)\right)^{p}+\varepsilon,
$$

for each $|t| \geq L$ and $j \in \mathbb{Z}[1, b]$.

Since $f(j, t)$ is continuous with respect to $t$, we also let

$$
\begin{aligned}
& m:=\min \{f(j, t):(j, t) \in \mathbb{Z}[1, b] \times[-L, L]\}, \\
& M:=\max \{f(j, t):(j, t) \in \mathbb{Z}[1, b] \times[-L, L]\} .
\end{aligned}
$$

Thus, when $t \geq L$, one follows

$$
\begin{aligned}
F(j, t)= & \int_{0}^{L} f(j, s) \mathrm{d} s+\int_{L}^{t} f(j, s) \mathrm{d} s \\
\geq & m L+\frac{1}{p}\left((b+1)^{1-\frac{p}{2}}\left(2 \sin \left(\frac{b \pi}{2(b+1)}\right)\right)^{p}+\varepsilon\right) \\
& \cdot\left(t^{p}-L^{p}\right) \\
= & \left(\frac{(b+1)^{1-(p / 2)}}{p}\left(2 \sin \left(\frac{b \pi}{2(b+1)}\right)\right)^{p}+\frac{\varepsilon}{p}\right) t^{p} \\
& -\left(\frac{(b+1)^{1-(p / 2)}}{p}\left(2 \sin \left(\frac{b \pi}{2(b+1)}\right)\right)^{p}+\frac{\varepsilon}{p}\right) L^{p}+m L .
\end{aligned}
$$

When $t \leq-L$, we have

$$
\begin{aligned}
F(j, t)= & -\left[\int_{t}^{-L} f(j, s) \mathrm{d} s+\int_{-L}^{0} f(j, s) \mathrm{d} s\right] \\
\geq & \frac{1}{p}\left((b+1)^{1-(p / 2)}\left(2 \sin \left(\frac{b \pi}{2(b+1)}\right)\right)^{p}+\varepsilon\right) \\
& \cdot\left((-t)^{p}-L^{p}\right)-M L \\
= & \left(\frac{(b+1)^{1-(p / 2)}}{p}\left(2 \sin \left(\frac{b \pi}{2(b+1)}\right)\right)^{p}+\frac{\varepsilon}{p}\right)(-t)^{p} \\
& -\left(\frac{(b+1)^{1-(p / 2)}}{p}\left(2 \sin \left(\frac{b \pi}{2(b+1)}\right)\right)^{p}+\frac{\varepsilon}{p}\right) L^{p}-M L .
\end{aligned}
$$

Put

$$
C=-\left(\frac{(b+1)^{1-(p / 2)}}{p}\left(2 \sin \left(\frac{b \pi}{2(b+1)}\right)\right)^{p}+\frac{\varepsilon}{p}\right) L^{p}+L \min \{m,-M\} .
$$

Therefore,

$$
F(j, t) \geq\left(\frac{(b+1)^{1-(p / 2)}}{p}\left(2 \sin \left(\frac{b \pi}{2(b+1)}\right)\right)^{p}+\frac{\varepsilon}{p}\right)|t|^{p}+C .
$$

Hence, our argument holds, and the proof is complete.

Remark 2. We observe that there are functions that satisfy $\left(F_{1}\right)$, but $\left(f_{1}\right)$ does not hold, see, such as example 1 in Section 4.

Similarly, we have the following result.

Corollary 2. Let $p \in[2,+\infty)$, for every $j \in \mathbb{Z}[1, b]$, $f(j, \cdot): \mathbb{R} \longrightarrow \mathbb{R}$ be continuous, and satisfy one of the following conditions:

$$
\begin{aligned}
& \left(f_{3}\right) \lim _{t \rightarrow \infty} \sup \frac{f(j, t)}{|t|^{p-2} t}<(b+1)^{1-(p / 2)}\left(2 \sin \left(\frac{\pi}{2(b+1)}\right)\right)^{p}, \quad \forall j \in \mathbb{Z}[1, b], \\
& \left(f_{4}\right) \lim _{t \rightarrow \infty} \inf \frac{f(j, t)}{|t|^{p-2} t}>b^{(p / 2)-1}\left(2 \sin \left(\frac{b \pi}{2(b+1)}\right)\right)^{p}, \quad \forall j \in \mathbb{Z}[1, b] .
\end{aligned}
$$


Then, problem (1) admits at least one solution.

The following results guarantee the existence of two nontrivial solutions to problem (1).

Theorem 5. Let $p \in(0,2)$, for every $j \in \mathbb{Z}[1, b]$, $f(j, \cdot): \mathbb{R} \longrightarrow \mathbb{R}$ be continuous, and $\left(F_{1}\right)$ holds. Additionally, assume that

$$
\left(\widetilde{F}_{1}\right) \lim _{t \rightarrow 0} \inf \frac{F(j, t)}{t^{2}}>2 \sin ^{2}\left(\frac{b \pi}{2(b+1)}\right), \quad \forall j \in \mathbb{Z}[1, b] .
$$

Then, problem (1) has at least two nontrivial solutions.

Proof. Our aim is to apply Lemma 2 . To this purpose, take $E=S$ and $I$ as in (11). Let $J(u):=-I(u)$, for all $u \in S$. Using Theorem 1 , it is evident that $J$ is anticoercive. Since the dimension of $S$ is finite, we can easily deduce that $J$ satisfies the P.S. condition. Next, we prove that $J$ verifies the mountain pass geometry.

From $\left(\widetilde{F}_{1}\right)$, there are $\varepsilon>0$ and $\delta_{1}>0$ such that

$$
F(j, t) \geq\left(2 \sin ^{2}\left(\frac{b \pi}{2(b+1)}\right)+\varepsilon\right) t^{2}=\left(\frac{\lambda_{b}}{2}+\varepsilon\right) t^{2}
$$

for every $(j, t) \in \mathbb{Z}[1, b] \times\left[-\delta_{1}, \delta_{1}\right]$.

By (8), Lemma 1 , and the inequality $(1+x)^{\theta} \leq 1+\theta x$, for $0<\theta \leq 1, x \geq 0$, we have

$$
\begin{aligned}
J(u) & =\sum_{j=1}^{b} F(k, u(j))-\frac{1}{p} \sum_{j=0}^{b}\left(\left(1+(\Delta u(j))^{2}\right)^{p / 2}-1\right) \\
& \geq\left(\frac{\lambda_{b}}{2}+\varepsilon\right) \sum_{j=1}^{b}(u(j))^{2}-\frac{1}{2} \sum_{j=0}^{b}(\Delta u(j))^{2} \\
& \geq \varepsilon\|u\|^{2},
\end{aligned}
$$

for $u \in S$ with $\|u\| \leq \delta_{1}$.

Writing $\alpha=\varepsilon \delta_{1}^{2}$ and $\rho=\delta_{1}$, then $J(u) \geq \alpha>0, \forall u \in \partial B_{\rho}$. Hence, $J$ satisfies condition (i) of Lemma 2. It is obvious that $J(0)=0$. Because $\lim _{\|u\| \longrightarrow+\infty} J(u)=-\infty$, we may pick $e \in S$ and $\|e\|>\rho$ with $J(e)<0$, which means $J$ satisfying condition (ii) of Lemma 2.

According to Lemma 2, $J$ has a critical value $c \geq \alpha$ given by $c=\inf _{\gamma \in \Gamma} \max _{u \in \gamma([0,1])} J(u)$, where

$$
\Gamma=\{\gamma \in C([0,1], S) \mid \gamma(0)=0, \gamma(1)=e\} \text {. }
$$

Set $u^{*} \in S$ be a critical point of $J$ and $J\left(u^{*}\right)=c \geq \alpha>0$, then $u^{*}$ is a nonzero solution of problem (1). By Theorem $1, J$ possesses a critical point $\bar{u} \in S$ with $J(\bar{u})=\sup _{u \in S} J(u)$. Noting that $J(\bar{u}) \geq J\left(u^{*}\right)>0$, we have $\bar{u} \neq 0$.

If $\bar{u} \neq u^{*}$, then the proof is complete.

If $\bar{u}=u^{*}$, we divide the following proof into two cases.
Case I. $b=1$. In such a case, we may see that $S=\{t \bar{u}: t \in \mathbb{R}\}$. Using $\lim _{\|u\| \rightarrow+\infty} J(u)=-\infty$ again, there exists $\tilde{t}<-\delta_{1} /\|\bar{u}\|$ satisfying $J(\tilde{t} \bar{u})<0$. Since $J(0)=0 \quad$ and $J\left(-\left(\delta_{1} /\|\bar{u}\|\right) \bar{u}\right)>0$, there exists $\bar{t} \in(\tilde{t}, 0)$ such that $J(\bar{t} \bar{u})=\max \{J(t \bar{u}), t \in[\widetilde{t}, 0]\}$. This shows that $\bar{t} \bar{u}$ is a critical point of $J$. Hence, problem (1) admits two nontrivial solutions.

Case II. $b \geq 2$. Through the definition of $u^{*}$, we show that $\max _{u \in \gamma([0,1])} J(u)=J(\bar{u})$ for every $\gamma \in \Gamma$. Considering that $b \geq 2$, there exists $d \in S$ which is linearly independent with $e$. For every $k \in \mathbb{N}$, define

$$
\gamma_{k}= \begin{cases}t(k d+e), & t \in\left[0, \frac{1}{2}\right), \\ (1-t) k d+t e, & t \in\left[\frac{1}{2}, 1\right],\end{cases}
$$

then $\gamma_{k} \in \Gamma$. Thus, we have $t_{k} \in(0,1)$ satisfying $J\left(\gamma_{k}\left(t_{k}\right)\right)=J(\bar{u})$. Obviously, $\gamma_{m}\left(t_{m}\right) \neq \gamma_{n}\left(t_{n}\right)$ when $m \neq n$, and $\left\{\gamma_{k}\left(t_{k}\right)\right\}$ is a sequence of pairwise distinct critical points of $J$ in $S$. Consequently, problem (1) has infinitely many nontrivial solutions. The proof is finished.

Theorem 6. Let $p \in[1,2)$, for every $j \in \mathbb{Z}[1, b]$, $f(j, \cdot): \mathbb{R} \longrightarrow \mathbb{R}$ be continuous, and $\left(F_{2}\right)$ holds. Moreover, suppose that

$$
\left(\widetilde{F}_{2}\right) \lim _{t \rightarrow 0} \sup \frac{F(j, t)}{t^{2}}<2 \sin ^{2}\left(\frac{\pi}{2(b+1)}\right), \quad \forall j \in \mathbb{Z}[1, b] .
$$

Then, problem (1) admits at least two nontrivial solutions.

Proof. Let $E=S$ and $I$ as in (11). Owing to Theorem 2, $I$ is anticoercive. Noting that $S$ is a finite dimensional space, it is obvious that $I$ satisfies the P.S. condition. Now, we show that $I$ satisfies the mountain pass geometry.

By $\left(\widetilde{F}_{2}\right)$, we pick $\varepsilon \in(0,1)$ such that

$$
\lim _{t \rightarrow 0} \sup \frac{F(j, t)}{t^{2}}<\frac{4(1-\varepsilon)}{(1+\varepsilon)^{p / 2}+1} \sin ^{2}\left(\frac{\pi}{2(b+1)}\right)=\frac{1-\varepsilon}{(1+\varepsilon)^{p / 2}+1} \lambda_{1},
$$

for each $j \in \mathbb{Z}[1, b]$.

Hence, there exists $\delta_{2}>0$, and we follow that

$$
F(j, t) \leq \frac{1-\varepsilon}{(1+\varepsilon)^{p / 2}+1} \lambda_{1} t^{2},
$$

when $(j, t) \in \mathbb{Z}[1, b] \times\left[-\delta_{2}, \delta_{2}\right]$.

Thanks to (8), for $u \in S$ with $\|u\| \leq \delta_{2}$, we get

$$
\Psi(u)=\sum_{j=1}^{b} F(j, u(j)) \leq \frac{(1-\varepsilon) \lambda_{1}}{(1+\varepsilon)^{p / 2}+1}\|u\|^{2} .
$$

For $u \in S$ with $\|u\| \leq\left(\varepsilon / \lambda_{b}\right)^{1 / 2}$, exploiting Lemma 1 , we get 


$$
\sum_{j=0}^{b}(\Delta u(j))^{2} \leq \lambda_{b}\|u\|^{2} \leq \varepsilon .
$$

By Lemma 1 and the inequality $(1+x)^{\theta} \geq$ $1+\theta x$, whenever $\theta \geq 1, x \geq 0$, we follow that

$$
\begin{aligned}
\Phi(u) & =\frac{1}{p} \sum_{j=0}^{b} \frac{\left(1+(\Delta u(j))^{2}\right)^{p}-1}{\left(1+(\Delta u(j))^{2}\right)^{p / 2}+1} \\
& \geq \frac{1}{p\left((1+\varepsilon)^{p / 2}+1\right)} \sum_{j=0}^{b}\left(\left(1+(\Delta u(j))^{2}\right)^{p}-1\right) \\
& \geq \frac{\lambda_{1}}{\left((1+\varepsilon)^{p / 2}+1\right)}\|u\|^{2},
\end{aligned}
$$

for $u \in S$ with $\|u\| \leq\left(\varepsilon / \lambda_{b}\right)^{1 / 2}$.

Let $\delta=\min \left\{\delta_{2},\left(\varepsilon \lambda_{b}^{-1}\right)^{1 / 2}\right\}$, then combining (56) and (58), we have

$$
\begin{aligned}
I(u) & \geq \frac{\lambda_{1}}{\left((1+\varepsilon)^{p / 2}+1\right)}\|u\|^{2}-\frac{(1-\varepsilon) \lambda_{1}}{(1+\varepsilon)^{p / 2}+1}\|u\|^{2} \\
& =\frac{\varepsilon \lambda_{1}}{(1+\varepsilon)^{p / 2}+1}\|u\|^{2},
\end{aligned}
$$

when $u \in S$ with $\|u\| \leq \delta$.

Picking $\alpha=\varepsilon \lambda_{1} \delta^{2} /\left((1+\varepsilon)^{p / 2}+1\right)$ and $\rho=\delta$, then for every $u \in \partial B_{\rho}, I(u) \geq \alpha>0$. The rest of the proof runs as the proof of Theorem 5 .

Theorem 7. Let $p \in[2,+\infty)$, for every $j \in \mathbb{Z}[1, b]$, $f(j, \cdot): \mathbb{R} \longrightarrow \mathbb{R}$ be continuous. Suppose that $\left(F_{3}\right)$ and $\left(\widetilde{F}_{1}\right)$ are satisfied. Then, problem (1) has at least two nontrivial solutions.

Proof. Take $E=S$ and $I$ as in (11) and denote $J(u):=-I(u)$, for all $u \in S$. Applying Theorem 3, we can derive that $J$ is anticoercive. Here, we only verify that $J$ satisfies condition (i) of Lemma 2.

Since $2 \leq p \leq+\infty$, there exists $n \in \mathbb{N}$ such that $n \leq(p / 2)<n+1$.

Using Taylor's formula, we have the following inequality:

$$
\left(1+x^{2}\right)^{p / 2}-1 \leq \frac{p}{2} x^{2}+\frac{p(p-2)}{2^{2} 2 !} x^{4}+\cdots+\frac{p(p-2) \cdot(p-2 n)}{2^{n+1}(n+1) !} x^{2 n+2} .
$$

From (9) and the above inequality, we follow that

$$
\begin{aligned}
\Phi(u) & =\frac{1}{p} \sum_{j=0}^{b}\left(\left(1+(\Delta u(j))^{2}\right)^{p / 2}-1\right) \\
& \leq \frac{1}{2} \sum_{j=0}^{b}(\Delta u(j))^{2}+\frac{p-2}{2^{2} 2 !} \sum_{j=0}^{b}(\Delta u(j))^{4}+\cdots+\frac{(p-2) \cdots(p-2 n)}{2^{n+1}(n+1) !} \sum_{j=0}^{b}(\Delta u(j))^{2 n+2} \\
& \leq \frac{1}{2} \sum_{j=0}^{b}(\Delta u(j))^{2}+\frac{p-2}{2^{2} 2 !}\left(\sum_{j=0}^{b}(\Delta u(j))^{2}\right)^{2}+\cdots+\frac{(p-2) \cdots(p-2 n)}{2^{n+1}(n+1) !}\left(\sum_{j=0}^{b}(\Delta u(j))^{2}\right)^{n+1} \\
& \leq \frac{\lambda_{b}}{2}\|u\|^{2}+\frac{p-2}{2 !}\left(\frac{\lambda_{b}}{2}\right)^{2}\|u\|^{4}+\cdots+\frac{(p-2) \cdots(p-2 n)}{(n+1) !}\left(\frac{\lambda_{b}}{2}\right)^{n+1}\|u\|^{2 n+2} .
\end{aligned}
$$

Owing to $\left(\widetilde{F}_{1}\right)$, there exist $\varepsilon>0$ and $\delta_{1}>0$ such that (49) is verified.

Because of the fact that

$\lim _{t \longrightarrow 0}\left[\frac{p-2}{2 !}\left(\frac{\lambda_{b}}{2}\right)^{2} t^{2}+\cdots+\frac{(p-2) \cdots(p-2 n)}{(n+1) !}\left(\frac{\lambda_{b}}{2}\right)^{n+1} t^{2 n}\right]=0$,

for the above $\varepsilon>0$, there is $\delta_{3}>0$ such that

$$
\frac{p-2}{2 !}\left(\frac{\lambda_{b}}{2}\right)^{2} t^{2}+\cdots+\frac{(p-2) \cdots(p-2 n)}{(n+1) !}\left(\frac{\lambda_{b}}{2}\right)^{n+1} t^{2 n} \leq \frac{\varepsilon}{2},
$$

whenever $|t| \leq \delta_{3}$.

Picking $\delta=\min \left\{\delta_{1}, \delta_{3}\right\}$, we have $\|u\|_{\infty} \leq\|u\| \leq \delta$ for all $u \in S$ with $\|u\| \leq \delta$.

Through (49), (61), and (63), we obtain 


$$
\begin{aligned}
J(u) & \geq\left(\frac{\lambda_{b}}{2}+\varepsilon\right)\|u\|^{2}-\frac{\lambda_{b}}{2}\|u\|^{2}-\frac{p-2}{2 !}\left(\frac{\lambda_{b}}{2}\right)^{2}\|u\|^{4}-\cdots-\frac{(p-2) \cdots(p-2 n)}{(n+1) !}\left(\frac{\lambda_{b}}{2}\right)^{n+1}\|u\|^{2 n+2} \\
& =\|u\|^{2}\left[\varepsilon-\frac{p-2}{2 !}\left(\frac{\lambda_{T}}{2}\right)^{2}\|u\|^{2}-\cdots-\frac{(p-2) \cdots(p-2 n)}{(n+1) !}\left(\frac{\lambda_{b}}{2}\right)^{n+1}\|u\|^{2 n}\right] \\
& \geq \frac{\varepsilon}{2}\|u\|^{2}
\end{aligned}
$$

for $u \in S$ with $\|u\| \leq \delta$.

Taking $\alpha=(\varepsilon / 2) \delta^{2}$ and $\rho=\delta$, we follow that $I(u) \geq \alpha>0$ if $u \in \partial B$. The proof is complete.

Applying the method analogous to that used above, we can draw the following conclusion.

Theorem 8. Let $p \in[2,+\infty)$, for every $j \in \mathbb{Z}[1, T]$, $f(j, \cdot): \mathbb{R} \longrightarrow \mathbb{R}$ be continuous. Suppose that $\left(F_{4}\right)$ and $\left(\widetilde{F}_{2}\right)$ are fulfilled. Then, problem (1) has at least two nontrivial solutions.

\section{Examples}

4.1. Example 1. Let $b=2, p=3 / 2$, and $f$ be a function which is defined as follows:

$$
\begin{aligned}
f(j, t)= & 2 j \sin \left(2 j t e^{t}\right)(1+t) e^{t}+\frac{21}{25}|t|^{1 / 2} \operatorname{sign}(t)\left[\sin \left(|t|^{3 / 2}\right)\right. \\
& \left.+|t|^{3 / 2} \cos \left(|t|^{3 / 2}\right)\right] .
\end{aligned}
$$

Since

$$
F(j, t)=\int_{0}^{t} f(j, s) \mathrm{d} s=2 \sin ^{2}\left(j t e^{t}\right)+\frac{14}{25}|t|^{3 / 2} \sin \left(|t|^{3 / 2}\right),
$$

through direct computations, we follow that

$$
\begin{gathered}
\lim _{|t| \longrightarrow \infty} \sup \frac{F(j, t)}{|t|^{3 / 2}}=\frac{14}{25}<\frac{2^{3 / 4}}{3}=\frac{2^{(3 / 4)-1}}{3 / 2}\left(2 \sin \left(\frac{\pi}{6}\right)\right)^{3 / 2}, \\
\lim _{t \rightarrow 0} \frac{F(j, t)}{t^{2}}=2 j^{2}>\frac{3}{2}=2 \sin ^{2}\left(\frac{\pi}{3}\right),
\end{gathered}
$$

for every $j \in \mathbb{Z}[1,2]$. Thus, the conditions $\left(F_{1}\right)$ and $\widetilde{F}_{1}$ are satisfied. Theorem 5 ensures that problem (1) admits at least two nontrivial solutions.

Moreover, we show that condition $\left(f_{1}\right)$ does not hold taking into account that

$$
\lim _{t \longrightarrow+\infty} \sup \frac{f(j, t)}{|t|^{(3 / 2)-2} t}=+\infty,
$$

for every $j \in \mathbb{Z}[1,2]$.
4.2. Example 2. When $p=4$, consider the following problem:

$$
\left\{\begin{array}{l}
-\triangle\left(\phi_{4, c}(\triangle u(j-1))\right)=35 j(u(j))^{3}+\frac{29}{50} u(j), \quad j \in \mathbb{Z}[1,3], \\
u(0)=u(4)=0 .
\end{array}\right.
$$

Noting that

$$
F(j, t)=\int_{0}^{t} f(j, s) \mathrm{d} s=\frac{35}{4} j t^{4}+\frac{29}{100} t^{2},
$$

we have

$$
\begin{aligned}
& \lim _{|t| \longrightarrow \infty} \inf \frac{F(j, t)}{t^{4}}=\frac{35}{4} j>\frac{3}{2}(\sqrt{2}+1)^{2}=\frac{3^{(4 / 2)-1}}{4}\left(2 \sin \left(\frac{3 \pi}{8}\right)\right)^{4}, \\
& \lim _{t \rightarrow 0} \sup \frac{F(j, t)}{t^{2}}=\frac{29}{100}<\frac{2-\sqrt{2}}{2}=2 \sin ^{2}\left(\frac{\pi}{8}\right),
\end{aligned}
$$

for every $j \in \mathbb{Z}[1,3]$. According to Theorem 8 , problem (69) has at least two nontrivial solutions.

\section{Data Availability}

Data sharing is not applicable to this article as no data sets were generated or analyzed during the current study.

\section{Conflicts of Interest}

The authors declare that they have no conflicts ofinterest.

\section{Authors' Contributions}

Both the authors contributed equally to the writing of this paper, and they read and approved the final manuscript.

\section{Acknowledgments}

This work was supported by the National Natural Science Foundation of China (Grant no. 11971126) and the Program for Changjiang Scholars and Innovative Research Team in University (Grant no. IRT_16R16). 


\section{References}

[1] Z. H. Chen and Y. T. Shen, "Infinitely many solutions of Dirichlet problem for $p$-mean curvature operator," Applied Mathematics-A Journal of Chinese Universities Series B, vol. 18, no. 2, pp. 161-172, 2003.

[2] P. De Nápoli and M. C. Mariani, "Mountain pass solutions to equations of $p$-Laplacian type," Nonlinear Analysis: Theory, Methods and Applications, vol. 54, no. 7, pp. 1205-1219, 2003.

[3] C. Bereanu and J. Mawhin, "Boundary value problems for second-order nonlinear difference equations with discrete $\phi$-Laplacian and singular $\phi$," Journal of Difference Equations and Applications, vol. 14, no. 10-11, pp. 1099-1118, 2008.

[4] D. Bonheure, P. Habets, F. Obersnel, and P. Omari, "Classical and non-classical solutions of a prescribed curvature equation," Journal of Differential Equations, vol. 243, no. 2, pp. 208-237, 2007.

[5] A. Cabada and V. Otero-Espinar, "Existence and comparison results for difference $\phi$-Laplacian boundary value problems with lower and upper solutions in reverse order," Journal of Mathematical Analysis and Applications, vol. 267, no. 2, pp. 501-521, 2002.

[6] J. Mawhin, "Periodic solutions of second order nonlinear difference systems with $\phi$-Laplacian: a variational approach," Nonlinear Analysis, vol. 75, pp. 4672-4687, 2012.

[7] R. P. Agarwal, Difference Equations and Inequalities, Theory, Methods, and Applications, Marcel Dekker Incorporated, New York, NY, USA, 2000.

[8] R. P. Agarwal and P. J. Y. Wong, Advanced Topics in Difference Equations, Kluwer Academic Publishers, Dordrecht, The Netherlands, 1997.

[9] S. Elaydi, An Introduction to Difference Equations, SpringerVerlag, New York, NY, USA, Third edition, 2011.

[10] W. G. Kelly and A. C. Peterson, Difference Equations: An Introduction with Applications, Academic Press, San Diego, CA, USA, 1991.

[11] Y. H. Long and L. Wang, "Global dynamics of a delayed twopatch discrete SIR disease model," Communications in Nonlinear Science and Numerical Simulation, vol. 83, Article ID 105117, 2020.

[12] J. S. Yu and B. Zheng, "Modeling Wolbachia infection in mosquito population via discrete dynamical model," Journal of Difference Equations and Applications, vol. 25, no. 11, pp. 1549-1567, 2019.

[13] Z. M. Guo and J. S. Yu, "Existence of periodic and subharmonic solutions for second-order superlinear difference equations," Science in China Series A: Mathematics, vol. 46, no. 4, pp. 506-515, 2003.

[14] P. Candito and G. D'Aguì, “Three solutions to a perturbed nonlinear discrete dirichlet problem," Journal of Mathematical Analysis and Applications, vol. 375, no. 2, pp. 594-601, 2011.

[15] G. D'Aguì, J. Mawhin, and A. Sciammetta, "Positive solutions for a discrete two point nonlinear boundary value problem with p-Laplacian," Journal of Mathematical Analysis and Applications, vol. 447, pp. 383-397, 2017.

[16] L. Erbe, B. G. Jia, and Q. Q. Zhang, "Homoclinic solutions of discrete nonlinear systems via variational method," Journal of Applied Analysis \& Computation, vol. 9, no. 1, pp. 271-294, 2019.

[17] L. Q. Jiang and Z. Zhou, "Three solutions to Dirichlet boundary value problems for advances in difference equations," Advances in Difference Equations, vol. 2008, no. 1, Article ID 345916, 2008.

[18] J. H. Kuang and Z. M. Guo, "Heteroclinic solutions for a class of p-Laplacian difference equations with a parameter," $A p$ plied Mathematics Letters, vol. 100, Article ID 106034, 2020.

[19] G. H. Lin and Z. Zhou, "Homoclinic solutions of discrete $\phi$-Laplacian equations with mixed nonlinearities," Communications on Pure and Applied Analysis, vol. 17, pp. 1723-1747, 2018.

[20] G. H. Lin, Z. Zhou, and J. S. Yu, "Ground state solutions of discrete asymptotically linear Schrodinger equations with bounded and non-periodic potentials," Journal of Dynamics and Differential Equations, vol. 32, no. 2, pp. 527-555, 2020.

[21] Y. H. Long and J. L. Chen, "Existence of multiple solutions to second-order discrete Neumann boundary value problems," Applied Mathematics Letters, vol. 83, pp. 7-14, 2018.

[22] Y. H. Long and S. H. Wang, "Multiple solutions for nonlinear functional difference equations by the invariants sets of descending flow," Journal of Difference Equations and Applications, vol. 25, no. 12, pp. 1768-1789, 2019.

[23] H. P. Shi, "Periodic and subharmonic solutions for secondorder nonlinear difference equations," Journal of Applied Mathematics and Computing, vol. 48, pp. 157-171, 2015.

[24] X. H. Tang, "Non-Nehari manifold method for periodic discrete super linear Schrodinger equation," Acta Mathematica Sinica, English Series, vol. 32, no. 4, pp. 463-473, 2016.

[25] Z. G. Wang and Z. Zhou, "Boundary value problem for a second-order difference equation with resonance," Complexity, vol. 2020, Article ID 7527030, 10 pages, 2020.

[26] Q. Q. Zhang, "Homoclinic orbits for a class of discrete periodic Hamiltonian systems," Proceedings of the American Mathematical Society, vol. 143, pp. 3155-3163, 2015.

[27] Q. Q. Zhang, "Homoclinic orbits for discrete Hamiltonian systems with indefinite linear part," Communications on Pure and Applied Analysis, vol. 14, no. 5, pp. 1929-1940, 2017.

[28] Q. Q. Zhang, "Homoclinic orbits for discrete Hamiltonian systems with local super-quadratic conditions," Communications on Pure and Applied Analysis, vol. 18, pp. 425-434, 2019.

[29] Z. Zhou and D. F. Ma, "Multiplicity results of breathers for the discrete nonlinear Schrodinger equations with unbounded potentials," Science China Mathematics, vol. 58, no. 4, pp. 781-790, 2015.

[30] Z. Zhou and M. T. Su, "Boundary value problems for Laplacian difference equations containing both advance and retardation," Applied Mathematics Letters, vol. 41, pp. 7-11, 2015.

[31] Z. Zhou and J. S. Yu, "“Homoclinic solutions in periodic nonlinear difference equations with superlinear nonlinearity," Acta Mathematica Sinica, English Series, vol. 29, no. 9, pp. 1809-1822, 2013.

[32] Z. Zhou, J. S. Yu, and Y. M. Chen, "Homoclinic solutions in periodic difference equations with saturable nonlinearity," Science China Mathematics, vol. 54, no. 1, pp. 83-93, 2011.

[33] P. Candito and G. M. Bisci, "Existence of two solutions for a second-order discrete boundary value problem," Advanced Nonlinear Studides, vol. 11, pp. 443-453, 2011.

[34] G. Bonanno, "A critical point theorem via the Ekeland variational principle," Nonlinear Analysis: Theory, Methods \& Applications, vol. 75, no. 5, pp. 2992-3007, 2012. 
[35] G. Bonanno and P. Candito, "Infinitely many solutions for a class of discrete non-linear boundary value problems," $A p$ plicable Analysis, vol. 88, no. 4, pp. 605-616, 2009.

[36] G. Bonanno, P. Candito, and G. D'Aguì, "Variational methods on finite dimensional Banach spaces and discrete problems," Advanced Nonlinear Studies, vol. 14, pp. 915-939, 2014.

[37] G. Bonanno, P. Jebelean, and C. Şerban, "Superlinear discrete problems," Applied Mathematics Letters, vol. 52, pp. 162-168, 2016.

[38] P. Candito, L. Li, and R. Livrea, "Infinitely many solutions for a perturbed nonlinear Navier boundary value problem involving the -biharmonic," Nonlinear Analysis: Theory, Methods \& Applications, vol. 75, no. 17, pp. 6360-6369, 2012.

[39] J. X. Ling and Z. Zhou, "Positive solutions of the discrete Dirichlet problem involving the mean curvature operator," Open Mathematics, vol. 17, no. 1, pp. 1055-1064, 2019.

[40] M. K. Moghadsm, S. Heidarkhani, and J. Henderson, "Infinitely many solutions for perturbed difference equations," Journal of Difference Equations and Applications, vol. 20, no. 7, pp. 1055-1068, 2014.

[41] S. A. Marano and D. Motreanu, "Infinitely many critical points of non-differentiable functions and applications to a Neumann type problem involving the $p$-Laplacian," Journal of Differential Equations, vol. 182, pp. 108-120, 2002.

[42] J. X. Wang and Z. Zhou, "Large constant-sign solutions of discrete Dirichlet boundary value problems with $p$-mean curvature operator," Mathematics, vol. 8, no. 3, p. 12, 2020.

[43] Z. Zhou and J. X. Ling, "Infinitely many positive solutions for a discrete two point nonlinear boundary value problem with $\phi_{c}$-Laplacian," Applied Mathematics Letters, vol. 91, pp. 28-34, 2019.

[44] G. H. Hardy, J. E. Littewood, and G. Pólya, Inequalities, Cambridge University Press, Cambridge, UK, Second edition, 1988.

[45] P. H. Rabinowitz, Minimax Methods in Critical Point Theory with Applications to Differential Equations, Vol. 65, American Mathematical Society, Providence, RI, USA, 1986. 